3-1-2013

\title{
Kerlan-Jobe Orthopaedic Clinic overhead athlete scores in asymptomatic professional baseball pitchers.
}

\author{
Matthew J Kraeutler \\ Thomas Jefferson University \\ Michael G Ciccotti \\ Thomas Jefferson University \\ Christopher C Dodson \\ Thomas Jefferson University \\ Robert W Frederick \\ Thomas Jefferson University \\ BoripowCammanarotadaitional works at: https://jdc.jefferson.edu/rothman_institute \\ Philadelphia Phillies \\ Part of the Orthopedics Commons \\ Let us know how access to this document benefits you
}

\section{Recommended Citation}

Kraeutler, Matthew J; Ciccotti, Michael G; Dodson, Christopher C; Frederick, Robert W;

Cammarota, Brian; and Cohen, Steven B., "Kerlan-Jobe Orthopaedic Clinic overhead athlete scores in asymptomatic professional baseball pitchers." (2013). Rothman Institute Faculty

Papers. Paper 33.

https://jdc.jefferson.edu/rothman_institute/33

This Article is brought to you for free and open access by the Jefferson Digital Commons. The Jefferson Digital Commons is a service of Thomas Jefferson University's Center for Teaching and Learning (CTL). The Commons is a showcase for Jefferson books and journals, peer-reviewed scholarly publications, unique historical collections from the University archives, and teaching tools. The Jefferson Digital Commons allows researchers and interested readers anywhere in the world to learn about and keep up to date with Jefferson scholarship. This article has been accepted for inclusion in Rothman Institute Faculty Papers by an authorized administrator of the Jefferson Digital Commons. For more information, please contact: JeffersonDigitalCommons@jefferson.edu. 


\section{Authors}

Matthew J Kraeutler, Michael G Ciccotti, Christopher C Dodson, Robert W Frederick, Brian Cammarota, and Steven B. Cohen 


\title{
As submitted to:
}

\section{Journal of shoulder and elbow surgery}

\section{And later published as:}

\section{Kerlan-Jobe Orthopaedic Clinic overhead athlete scores in asymptomatic professional baseball players.}

\author{
March 2013, Volume 22, Issue 3, pp. 329-32
}

doi: 10.1016/j.jse.2012.02.010

\begin{abstract}
Background

The Kerlan-JobeOrthopaedic Clinic (KJOC) Shoulder and Elbow score is a subjective questionnaire that has been validated and been shown to be more specific in overhead athletes than the American Shoulder and Elbow Society (ASES) scale. The purpose of this study was to determine a mean and reasonable range of KJOC scores within which a healthy asymptomatic professional baseball pitcher will fall. It was hypothesized that healthy professional baseball pitchers would have very high KJOC scores (>90 out of 100 possible points).
\end{abstract}


KJOC questionnaires were given to all healthy pitchers before the start of the season at all levels in one professional minor league system. Pitchers were asked to complete the questionnaire upon reporting to their AAA, AA, or A affiliate team. Any pitcher starting the season on the disabled list was excluded from the study.

Results

KJOC scores were returned by 44 pitchers. Average score for all pitchers was 94.82 ( $95 \% \mathrm{Cl}$ 92.94-96.70). Average score for each question was greater than 9 out of 10 . The average score for the AAA affiliate was significantly higher than that for the AA affiliate $(p=0.015)$. No other significant differences in scores were found between class-levels or groups based on professional playing experience.

\section{Conclusion}

Only 7 out of $44(16 \%)$ healthy asymptomatic pitchers had a KJOC score below 90 . Therefore, we believe that the KJOC score is an accurate assessment for overhead athletes and normal values should be greater than 90 . Anything below this value could be a potential cause for concern for team physicians.

Level of Evidence: IV

Keywords: KJOC score; asymptomatic; professional baseball pitcher; overhead athlete 


\section{Introduction}

The Kerlan-Jobe Orthopaedic Clinic (KJOC) Overhead Athlete Shoulder and Elbow score was initially developed in 2010 as a useful shoulder and elbow questionnaire to supplement existing validated questionnaires but designed specifically for overhead athletes. It has been validated with the Disabilities of the Arm, Shoulder and Hand (DASH) score as well as the DASH sports/performing arts module ${ }^{1,2}$ and has been shown to be more sensitive in overhead athletes than the American Shoulder and Elbow Society (ASES) scale ${ }^{4}$. In addition, the KJOC score has been shown to effectively distinguish between professional baseball players playing without pain, playing with pain, and not playing due to pain. $^{1,2}$

The KJOC questionnaire has also been used to evaluate Little League baseball players with recent medial-sided elbow pain with overhead throwing. ${ }^{5}$ An average score of 60.3 out of 100 was measured for these players. In another study, professional or collegiate overhead athletes at least one year following superior labral anterior posterior (SLAP) lesion repair filled out a KJOC questionnaire with a mean score of 76.9 (range 63.5-92.5). ${ }^{3}$ Finally, a study comprising an assortment of overhead athletes after SLAP lesion repair measured an average KJOC score of 73.6 (range 39-100) at an average follow-up time of 3.5 years. ${ }^{4}$ However, no study has measured the KJOC scores of healthy overhead athletes.

The purpose of this study was to determine a mean and reasonable range of KJOC scores within which a healthy professional baseball pitcher will fall. We hypothesized that healthy overhead athletes should score greater than 90 points and this score could serve as a baseline for future evaluations of professional pitchers to determine how significant a particular injury may be. 


\section{Materials and Methods}

Approval for this study was obtained from the Institutional Review Board (IRB). KJOC questionnaires were given to healthy pitchers in one professional baseball minor league system at the start of the 2011 season. A healthy pitcher was defined as any pitcher starting the regular season at their assigned minor league level who did not have any acute injury affecting complete unrestricted participation in baseball. All athletes underwent a preseason physical examination.

All pitchers were asked to complete the questionnaire following completion of spring training but before the start of the season. All KJOC scores were then tabulated. An overall average score for the entire study group as well as scores for each minor league level and professional baseball experience were determined.

Because we were unable to find any previously published studies on $\mathrm{KJOC}$ scores exclusively in healthy professional baseball pitchers, we did not feel it would be appropriate to perform a power analysis prior to study initiation. Analysis of variance (ANOVA) and student's t-tests were performed to determine significant differences in $\mathrm{KJOC}$ scores between groups based on minor league level and professional playing experience. Confidence intervals were calculated by average score $+/-t^{*}(\sigma / \sqrt{n})$. A p-value less than 0.05 was considered statistically significant. 
Results

KJOC scores were given to and completed by all 44 pitchers (100\%) assigned to the minor league teams. The average score for all pitchers was 94.82 (95\% Cl 92.94-96.70, range 75-100). The average score for each question was greater than 9 out of 10 (Table 1). Average scores for the AAA, AA, High-A, and Low-A affiliates were 97.73 (95\% Cl 95.41-100.04), 93.33 (95\% Cl 90.47-96.19), 94.44 (95\% Cl 88.06-100.83), and 93.92 (95\% Cl89.15-98.68), respectively (Table 2). Using a one-way analysis of variance (ANOVA) test, no significant difference was found across groups $(p=0.34)$. However, a student's t-test showed that the average score for the AAA affiliate was significantly higher than that for the AA affiliate $(p=0.015)$. No other significant differences in scores were found between class levels using a student's t-test.

Pitchers were also divided by the number of years of professional playing experience (Table 3). Overall, the average number of years of experience was 4.0 (range, 1-13 years). Players with the most experience (>5 years) had the highest scores, while players with 2-3 years of experience had the lowest scores. Using a one-way ANOVA test, no significant difference in scores was found across the groups $(p=0.28)$. Furthermore, no significant difference was found between any two groups using a student's t-test. 
KJOC scores asymptomatic professional baseball pitchers

\section{Discussion}

The Kerlan-Jobe Orthopaedic Clinic Overhead Athlete Shoulder and Elbow score is an effective tool for assessing the performance and function of overhead athletes, particularly baseball pitchers. We have shown in this study that healthy professional baseball pitchers have very high KJOC scores (total average $=94.8$ ). Their scores were much higher than those of $(1)$ Little League baseball players with medial-sided elbow pain with overhead throwing $(\Delta=34.5)^{5}$,(2) professional or collegiate overhead athletes at least one year following superior labral anterior

posterior (SLAP) lesion repair $(\Delta=17.9)^{3}$, and (3) a mixture of overhead athletes an average of 3.5 years following SLAP lesion repair $(\Delta=21.2)^{4}$.

While one cannot judge the truthfulness of the players completing the survey, 7 of them did have scores below 90 indicating their true response to their ability to pitch. We specifically asked the pitchers to complete the survey after they were assigned to their specific minor league level and had made the roster, in an effort to obtain the most accurate information. In addition, the specific responses were not communicated with the organization in order to maintain player privacy.

As noted above, 7 out of 44 (16\%) pitchers in our study had a score below 90. Therefore, we believe that an appropriate baseline score for a healthy professional baseball pitcher should be in the 90 s, confirming our hypothesis. A KJOC score below 90 should alert team physicians that a pitcher may be playing with an injury or pain or at least sense that he is not functioning at full capacity. However, two of the pitchers in our study had scores of 75 and 77 , respectively. Of the seven pitchers with scores below 90, only two (2/7, 29\%) were treated for any shoulder symptoms during the season. Both of these players were diagnosed with mild tendonitis of the rotator cuff or biceps tendon approximately 
KJOC scores asymptomatic professional baseball pitchers

three months into the season. Both responded to nonoperative treatment without missing significant playing time. Therefore, although average scores should be in the 90s for healthy professional baseball pitchers, this will not be the case for each individual. Team physicians should consider having baseline KJOC scores for each pitcher, particularly professional and collegiate players. This would potentially allow for the identification of an occult injury and give a comparison value should a pitcher become injured.

From our analysis, it seems that KJOC scores for professional baseball pitchers are not based on the number of years of professional playing experience. All groups based on experience had an average score greater than 90 , with the highest scores presented by the most experienced group ( $>5$ years experience).

Strengths of this study include the high response rate (100\%) and the incorporation of all four minor league levels of a professional baseball organization. This allowed us to show that there was a very consistent, narrow range of scores between each level of play. The weaknesses of this study also should be noted. Although all pitchers in this study were asymptomatic when completing the survey, it is not necessarily true that all pitchers were playing without pain. It is certainly possible and likely that some athletes may minimize or downplay any injury or pain for fear of advancement in the professional system. However, prior to assignment to any minor league level all players must have completed spring training and assessment of performance by members of the coaching staff and front office. Therefore, the purpose of this study was not to distinguish between pitchers with/without pain, but rather to determine an appropriate range of KJOC scores for "healthy" (asymptomatic) professional pitchers. Further studies should be done to determine if these baseline scores can be effectively compared to scores at a later time to identify occult injuries. In addition, we showed a significant difference in scores between AAA and AA players and thus there is the possibility of a significant difference between minor league and major league KJOC scores. Thus, there is the possibility that our 
KJOC scores asymptomatic professional baseball pitchers

reported average score may be slightly different than the 'true' score for all healthy professional baseball pitchers. Nevertheless, we believe the score we report is a very close estimate and can serve a useful purpose for team physicians. 
KJOC scores asymptomatic professional baseball pitchers

\section{Conclusions}

KJOC scores for healthy professional baseball pitchers are very high. However, scores are not based on class-level or professional playing experience. Thus, team physicians should not assess a pitcher's KJOC score based on these demographics. In addition, while scores are generally high for professional pitchers, some healthy pitchers will have lower scores. Therefore, the most effective way of assessing a pitcher's injury or general soreness may be to look at differences between the individual's 'healthy' score and his score following injury. However, further study would be required to determine a correlation between $\mathrm{KJOC}$ scores and a professional baseball pitcher's health status. 


\section{References}

1.Alberta FG, ElAttrache NS, Bissell S, Mohr K, Browdy J, Yocum L et al. The development and validation of a functional assessment tool for the upper extremity in the overhead athlete.Am J Sports Med2010;38(5):903-911. doi:10.1177/0363546509355642

2.Domb BG, Davis JT, Alberta FG, Mohr KJ, Brooks AG, ElAttrache NS et al. Clinical follow-up of professional baseball players undergoing ulnar collateral ligament reconstruction using the new Kerlan-JobeOrthopaedic Clinic overhead athlete shoulder and elbow score (KJOC Score).Am J Sports Med 2010;38(8):1558-1563. doi:10.1177/0363546509359060

3.Neri BR, EIAttrache NS, Owsley KC, Mohr K, Yocum LA. Outcome of type II superior labral anterior posterior repairs in elite overhead athletes: Effect of concomitant partial-thickness rotator cuff tears.Am J Sports Med2011;39(1):114-120. doi:10.1177/0363546510379971

4.Neuman BJ, Boisvert CB, Reiter B, Lawson K, Ciccotti MG, Cohen SB. Results of arthroscopic repair of type II superior labral anterior posterior lesions in overhead athletes: assessment of return to preinjury playing level and satisfaction. Am J Sports Med 2011;39(9):1883-

1888.doi:10.1177/0363546511412317

5.Wei AS, Khana S, Limpisvasti O, Crues J, Podesta L, Yocum LA. Clinical and magnetic resonance imaging findings associated with Little League elbow. J PediatrOrthop 2010;30(7):715-719. doi:10.1097/BPO.0b013e3181edba46 
Table Legends

1. Scores for each KJOC question. Average scores and a range are given for each KJOC question.

2. KJOC scores by minor league level.Average scores for players grouped by minor league playing level.

3. KJOC scores by experience level. Average scores for players grouped by the number of years of professional playing experience. 